\title{
Efficacy and Safety of Local Radiotherapy to All Oligometastatic Sites in Elderly Patients with Metachronous Oligometastatic Cancers After Initial Treatment for the Primary Tumor
}

\author{
Xiaolong $\mathrm{Hu}\left(\mathbb{D}^{\prime}\right.$ \\ Hongqi $\mathrm{Li}^{2}$ \\ Xiaoli Kang ${ }^{2}$ \\ Xuan Wang ${ }^{2}$ \\ Haifeng Pang ${ }^{2}$ \\ Chen Liu ${ }^{2}$ \\ Jianchun Zhang' \\ Yingjie Wang ${ }^{2}$ \\ 'Department of Radiation Oncology, \\ Beijing Geriatric Hospital, Beijing, \\ People's Republic of China; ${ }^{2}$ Department \\ of Radiation Oncology, Air Force General \\ Hospital, Beijing, People's Republic of \\ China
}

Background and Purpose: This study aimed to investigate the efficacy and safety of maintenance therapy combined with local radiotherapy at all oligometastatic sites (LRTOS) in elderly patients with metachronous oligometastatic cancers (MOC).

Patients and Methods: A total of 242 elderly patients with MOC ( $\leq 5$ metastases) and primary tumor well controlled after definitive treatment was retrospectively analyzed between August 2014 and February 2020 at Beijing Geriatric Hospital and Air Force General Hospital. Patients were divided into maintenance therapy group (maintenance therapy alone) and local radiotherapy group (maintenance therapy combined with LRTOS). Results: There were 86 patients in the local radiotherapy group and 156 patients in the maintenance therapy group. The median length of follow-up was 36 months (range, 8.0-62 months). Median overall survival (mOS) was 25 months (95\% CI: 21.1-28.9) in the local radiotherapy group and 16 months $(95 \%$ CI: $14.5-17.6)$ in the maintenance therapy group $(\mathrm{p}<0.001)$. Multivariate analyses demonstrated that LRTOS (hazard ratio $(\mathrm{HR})=0.49,95 \%$ confidence interval $(\mathrm{CI})$ : 0.35 0.67, $\mathrm{p}<0.001$ ), good Eastern Cooperative Oncology Group Performance Status (ECOG PS, HR = $0.69,95 \%$ CI: $0.49-0.97, p=0.032$ ), longer duration between diagnosis of primary tumor and occurrence of progression $(\mathrm{HR}=0.87,95 \% \mathrm{CI}$ : $0.78-0.97, \mathrm{p}=0.015)$, and subsequent systemic treatment $(\mathrm{HR}=0.52,95 \% \mathrm{CI}: 0.38-0.72, \mathrm{p}<0.001)$ were independent predictors of good OS. In patients who did not receive subsequent systemic treatment, their mOS was 21 months $(95 \% \mathrm{CI}$ : 12.8-29.2) for those treated with LRTOS and 14 months (95\% CI: 11.4-16.6) for those who did not receive local radiotherapy $(\mathrm{p}=0.001)$. Further multivariate analysis showed that LRTOS was the only independent factor for predicting good $\mathrm{OS}(\mathrm{HR}=0.47,95 \% \mathrm{CI}: 0.26-0.83, \mathrm{p}=0.010)$. Patients with metachronous oligometastatic lung cancer, colorectal cancer, prostate cancer, and breast cancer had higher survival benefits following LRTOS. Most patients suffered from grade 1-2 toxicities, but no treatment-related death was recorded.

Conclusion: This retrospective study shows that elderly patients with MOC treated with LRTOS may have better survival outcomes.

Keywords: elderly patients, local radiotherapy, metachronous oligometastatic cancers, residual disease

\section{Introduction}

Department of Radiation Oncology, Air Force General Hospital, Beijing, People's Republic of China

Tel +86-1068434886

Email Wangyj9999@I26.com
The prognosis of elderly patients with metastatic tumors is highly heterogeneous. ${ }^{1,2}$ These patients are less likely to receive guideline-recommended standard systemic treatment, and this may explain the poor prognosis. ${ }^{3}$ Local radiotherapy is applied 
as a palliative treatment for elderly patients with metastatic tumors to alleviate symptoms or prevent complications. However, factors such as underlying diseases, organ function decline, decreased self-repair ability, and geriatric syndrome reduce the efficacy of treatments for metastatic tumors in elderly patients. ${ }^{4}$

In 1995, Hellman and Weichselbaum ${ }^{5}$ first proposed the concept of "oligometastatic disease" as an intermediate state of cancer spread between localized disease and widespread metastases. Currently, oligometastases are defined as the presence of 3-5 metastases, ${ }^{6}$ and oligometastatic disease is classified into synchronous state (maximum sixmonth interval between primary tumor diagnosis and appearance of metastases) and metachronous state (more than six-month interval between primary tumor diagnosis and appearance of metastases). ${ }^{7-10}$ Several high-quality randomized controlled studies have shown that radical local therapy for primary tumor and all metastases can significantly improve the prognosis of patients with synchronous oligometastatic cancer. ${ }^{1-13}$ However, other reports have indicated that synchronous and metachronous states are two distinct diseases with different clinical behavior and prognosis. ${ }^{14-16}$ Palma et $\mathrm{al}^{17}$ reported that application of stereotactic ablative radiotherapy combined with standard-of-care systemic therapy in patients with metachronous oligometastatic cancer with controlled primary tumor improved the overall survival.

Herein, we retrospectively analyzed the efficacy and safety of maintenance therapy combined with local radiotherapy at all oligometastatic sites (LRTOS) in elderly patients with metachronous oligometastatic cancers (MOC).

\section{Patients and Methods}

\section{Patients}

A total of 242 elderly patients diagnosed with MOC at Beijing Geriatric Hospital and Air Force General Hospital between August 2014 and February 2020 were retrospectively analyzed. The inclusion criteria were patients aged $\geq 65$ years, with an Eastern Cooperative Oncology Group Performance Status (ECOG PS) score of $\leq 2$, histologically diagnosed with primary tumor, the interval duration from the initial diagnosis to metastasis $\geq 6$ months, MOC ( $\leq 5$ metastases) confirmed by comprehensive imaging examination (including brain magnetic resonance imaging (MRI) + whole-body positron emission tomography-computed tomography (PET-CT) or brain MRI + thoracic/abdominal/pelvic CT, and bone scan), and with the number of lesions per organ $\leq 3$. In all patients, no surgery, palliative radiotherapy, or radiofrequency ablation were performed to treat metastatic lesions, and the tumor boundary was clear. The definition and classification of metachronous oligometastatic disease was carried out in line with the European Society for Radiotherapy and Oncology and the European Organization for the Research and Treatment of Cancer (ESTRO-EORTC) consensus recommendation. ${ }^{10}$ For patients in which the diagnosis of metastases was not clear, a biopsy was carried out. All patients received definitive treatment which successfully controlled the primary tumors. Patients with pulmonary failure, cardiac failure, hepatic failure, renal failure, and hematologic tumor were excluded from the analysis.

Maintenance therapy consisted of systemic therapy (chemotherapy, molecular targeted therapy, immunotherapy, and endocrine therapy) and palliative symptomatic supportive treatment. Local radiotherapy techniques included three-dimensional conformal radiotherapy (3DCRT), intensity-modulated radiation therapy (IMRT), stereotactic body radiotherapy (SBRT), and stereotactic radiosurgery (SRS). Patients who received maintenance therapy alone were assigned into the maintenance therapy group, whereas those who received maintenance therapy combined with LRTOS were assigned into the local radiotherapy group.

The primary endpoint was OS, defined as the time from diagnosis of metastatic disease to the date of death. The study was conducted in line with the Declaration of Helsinki, as revised in 2013, and was approved by the Ethics Committee of Beijing Geriatric Hospital. Patients were included in this study only after obtaining written informed consent. All patients were followed up by telephone or during clinical visits.

\section{Local Radiotherapy}

In the local radiotherapy group, all patients were comprehensively examined before application of radiotherapy at all oligometastatic sites. Several factors such as age, cardiopulmonary function, underlying diseases, risk of pathological fractures, nutritional status, central nervous system symptoms, and risk-benefit ratio were assessed. For some patients, consultation with the relevant departments does not occur simultaneously. Radiation oncologists determined the local radiotherapy regimen based on the general patient condition, tumor location, size, boundary, pulmonary function, bone marrow, and hepatic and renal function. 
The radiotherapy regimen adopted was 40-70 Gy in 20-35 fractions, hypofractionated radiotherapy of 60-70 Gy in 15 fractions (the biologically effective dose $\left(\mathrm{BED}_{10}\right)$ was $84-103$ Gy using the linear-quadratic model with $\alpha / \beta$ $=10 \mathrm{~Gy})$, SBRT was $120-140 \mathrm{~Gy}\left(\mathrm{BED}_{10}\right)$, and SRS was $50 \%$ isodose line 22 Gy in 1 fraction (the head gamma knife). Isolated brain metastases up to $4.5 \mathrm{~cm}$ in size were treated with SRS. Lung metastases were treated with conventional fractionated radiotherapy if the tumor was near the esophagus. Conventional radiotherapy plus wholebrain radiation therapy (WBRT) were applied to three brain metastases (50 Gy in 20 fractions plus WBRT 40 Gy in 20 fractions or WBRT 30 Gy in 10 fractions alone). Conventional fractionated radiotherapy was applied for metastasis to superficial lymph nodes. For patients at risk of vertebral fracture (as determined by an orthopedic surgeon), such vertebrae were fixed before local radiotherapy.

\section{Toxicity Assessment}

Acute and long-term toxicity were graded before and after 90 days following treatment according to the National Cancer Institute (NCI) common terminology criteria for adverse events (CTCAE) version 5. Systemic therapyrelated acute toxicities included skin rash, diarrhea, nausea, leukopenia, fatigue, vomiting, and hepatic function impairment. The radiotherapy-related acute toxicities examined included, pneumonia, fatigue, thrombocytopenia, anemia, leukopenia, dermatitis, and esophagitis. For each type of toxicity, the patient was scored according to the highest grade of toxicity. The toxicity information for hospitalist patients was obtained from hospital records. Toxicity information for non-hospitalized patients was obtained from outpatient data records or through telephone follow-ups.

\section{Statistical Analysis}

All statistical analyses were performed using IBM SPSS software version 25 (SPSS Inc., Chicago, IL, USA) and GraphPad Prism 7 version 7.04 (GraphPad Software, Inc., La Jolla, California, USA). Descriptive data with normal distribution were expressed as mean \pm standard deviation (SD). Variables with skewed distribution were presented as the median and interquartile range (IQR). Frequency distributions and percentages were used to describe categorical variables. Kaplan-Meier analysis and the Log rank test were used to analyze survival curves. Univariable and multivariable analyses were conducted using the Cox proportional hazard model to assess possible prognostic factors and calculate survival hazard ratios (HRs) with 95\% confidence interval $(95 \% \mathrm{CI})$ for OS. Variables found to be significant in univariate analysis $(\mathrm{p}<0.05)$ were incorporated into multivariate Cox regression analysis to determine independent prognostic factors. This multivariate analysis was carried out using the backward step method. A two-sided p-value of $<0.05$ was considered statistically significant in all tests.

\section{Result \\ Patient Characteristics}

Patient inclusion and exclusion criteria are shown in Figure 1. Overall, 35.5\% $(\mathrm{n}=86)$ received LRTOS (local radiotherapy group) and $64.5 \%(\mathrm{n}=156)$ received maintenance therapy only (maintenance therapy group). The median age was 70 years, with $24.4 \%(n=59)$ of patients being older than 75 years. The male-to-female ratio was 1.74:1 (139 male patients, 103 female patients). Most patients had a junior high school education level or below ( $n=226,93.4 \%$ ). The distribution of patients according to the site of original primary tumor was as follows: colorectal cancer $(\mathrm{n}=60,24.8 \%)$, breast cancer $(\mathrm{n}=53,21.9 \%)$, prostate cancer $(\mathrm{n}=52,21.5 \%)$, lung cancer $(\mathrm{n}=51,21.1 \%)$, cervical cancer $(\mathrm{n}=18.7,40 \%)$, and other cancers $(\mathrm{n}=8,3.30 \%)$. The median time from diagnosis of the primary tumor to progression was 2.5 years. The distribution of patients according to oligometastasis was as follows: bone $(\mathrm{n}=133,55 \%)$, lung $(\mathrm{n}=79$, $32.6 \%)$, brain $(\mathrm{n}=56,23.1 \%)$, liver $(\mathrm{n}=56,23.1 \%)$, adrenal $(\mathrm{n}=13,5.40 \%)$, and non-region lymph nodes (n $=16,6.6 \%$ ). Ten patients performed pathological biopsy at oligometastatic sites when the diagnosis was not clear (non-regional lymph nodes, $\mathrm{n}=7$; lung, $\mathrm{n}=1$; adrenal, $\mathrm{n}=1$; and liver, $\mathrm{n}=1)$. Most patients $(68.2 \%, \mathrm{n}=165)$ received subsequent systemic treatment (chemotherapy, $\mathrm{n}=65$, molecular targeted therapy, $\mathrm{n}=38$, endocrine therapy, $\mathrm{n}=40$, and immunotherapy $=22$ ). The number of metastases was $1-2(n=145,59.9 \%)$ and $3-5(n=97$, $40.1 \%$ ) and the number of underlying diseases was $0-1$ (n $=103,42.6 \%), 2-3(\mathrm{n}=127,52.5 \%)$, and $4-5(\mathrm{n}=12$, $5.0 \%$ ). The distribution of TNM stage was as follows: stage I $(\mathrm{n}=17,7.0 \%)$, stage II $(\mathrm{n}=84,34.7 \%)$, and stage III $(\mathrm{n}=141,58.3 \%)$. Approximately $70 \%(\mathrm{n}=$ 170) of patients underwent PET-CT examination. Patient and disease characteristics of the two cohorts are summarized in Table 1. 


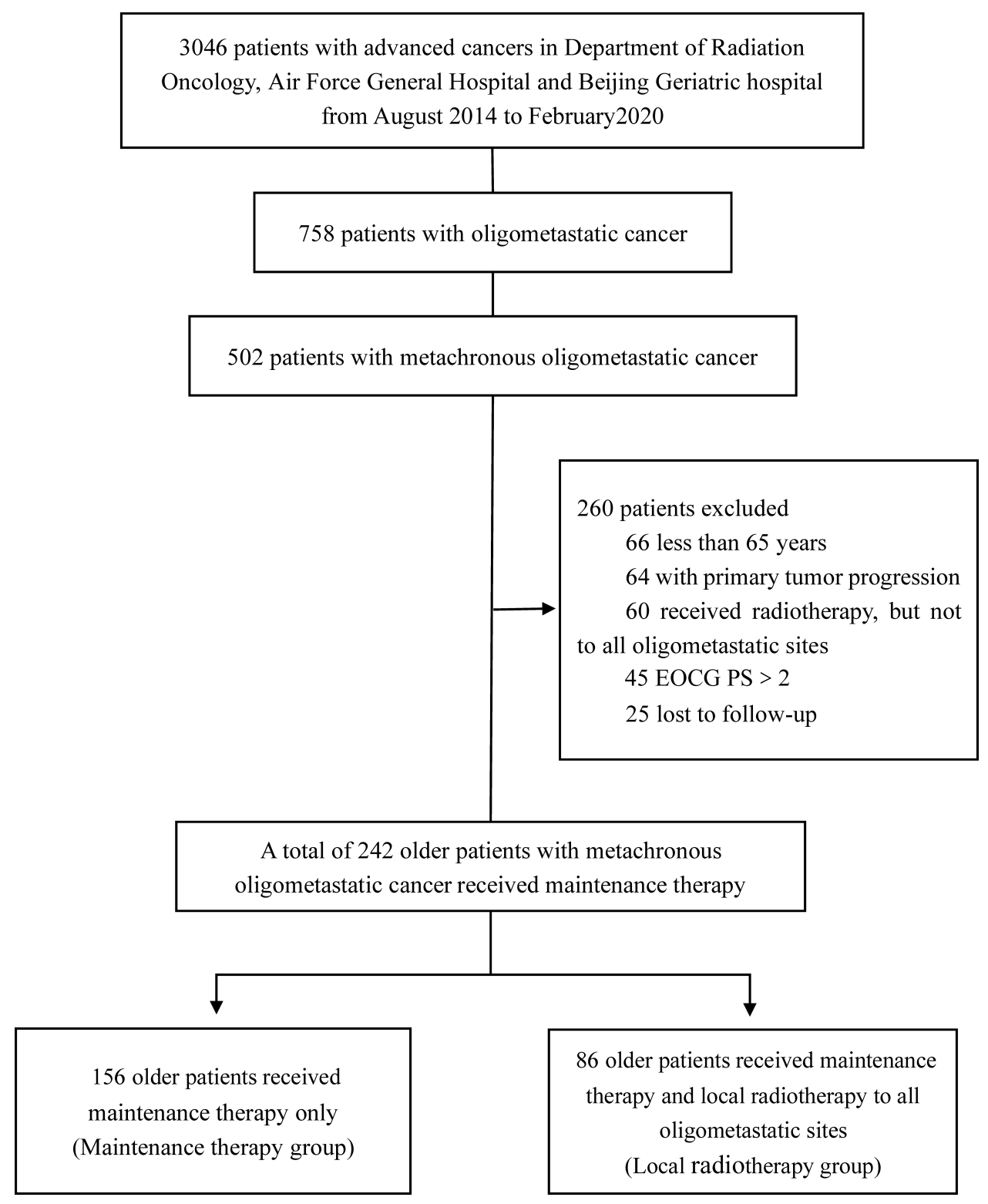

Figure I A flowchart of the study.

Abbreviation: ECOG PS, Eastern Cooperative Oncology Group Performance Status.

In the local radiotherapy group, 8 patients with solitary brain metastases were treated with 22 Gy in 1 fraction, 9 patients received WBRT, 7 patients with non-vertebral metastases received 60 Gy in 20 fractions (rib, $\mathrm{n}=2$; sternum, $\mathrm{n}=2$; humerus, $\mathrm{n}=1$; ilium, $\mathrm{n}=1$; and pubic, $\mathrm{n}=1$ ), 2 patients with a high risk of vertebral compression fracture underwent surgical fixation followed by local radiotherapy, 18 patients received SBRT for lung metastases $\left(\mathrm{BED}_{10}>100 \mathrm{~Gy}\right)$ (central type, $\mathrm{n}=5$; peripheral type, $\mathrm{n}=13), 8$ patients received body gamma knife to liver metastases (diameter $\leq 3.5 \mathrm{~cm}, \mathrm{n}=6$; diameter $>3.5 \mathrm{~cm}, \mathrm{n}=2$ ), 5 patients received SBRT for adrenal metastases, and 4 patients received conventional radiotherapy for non-region lymph nodes metastases. Local radiotherapy regimens applied are shown in Table 2.

\section{Survival Outcomes}

The median length of follow-up was 36 months (range, 8.062 months). The median overall survival (mOS) was 18 months (95\% CI: 16.3-19.7) for the entire cohort, 25 months (95\% CI: 21.1-28.9) for the local radiotherapy group, and 16 months (95\% CI: 14.5-17.6) for the maintenance therapy group ( $\mathrm{p}<0.001$, Figure 2$)$. In the subgroup of patients without subsequent systemic treatment, the mOS was 21 
Table I Patient Characteristics of Elderly Patients with Metachronous Oligometastatic Cancers

\begin{tabular}{|c|c|c|}
\hline & $\begin{array}{l}\text { Maintenance } \\
\text { Therapy } \\
(n=156)\end{array}$ & $\begin{array}{l}\text { Local } \\
\text { Radiotherapy } \\
(n=86)\end{array}$ \\
\hline Characteristics & No. (\%) & No. (\%) \\
\hline \multicolumn{3}{|l|}{ Gender } \\
\hline Male & $88(56.4 \%)$ & $5 \mathrm{I}(59.3 \%)$ \\
\hline Female & $68(43.6 \%)$ & 35 (40.7\%) \\
\hline Age & $70(67-76)$ & $69(66-72)$ \\
\hline \multicolumn{3}{|l|}{ EOCG performance status } \\
\hline $0-1$ & 92 (59.0\%) & $62(72.1 \%)$ \\
\hline 2 & $64(41.0 \%)$ & 24 (27.9\%) \\
\hline \multicolumn{3}{|l|}{ Smoking status } \\
\hline Nonsmoker & $80(51.3 \%)$ & $36(41.9 \%)$ \\
\hline Present or former smoker & $76(48.7 \%)$ & $50(58.1 \%)$ \\
\hline \multicolumn{3}{|l|}{ Educational degree } \\
\hline Elementary school & $5 \mathrm{I}(32.7 \%)$ & $19(22.1 \%)$ \\
\hline $\begin{array}{l}\text { Junior or senior high } \\
\text { school }\end{array}$ & $96(61.5 \%)$ & $60(69.8 \%)$ \\
\hline Undergraduate & $9(5.80 \%)$ & $7(8.10 \%)$ \\
\hline \multicolumn{3}{|l|}{ Underlying disease } \\
\hline Hypertension & $72(46.2 \%)$ & $38(44.2 \%)$ \\
\hline Diabetes & $52(33.3 \%)$ & $25(29.1 \%)$ \\
\hline Coronary heart disease & $63(40.4 \%)$ & 27 (3I.4\%) \\
\hline COPD & 31 (19.9\%) & 27 (3I.4\%) \\
\hline Atrial fibrillation & $58(37.4 \%)$ & $29(33.7 \%)$ \\
\hline \multicolumn{3}{|l|}{ No. of underlying disease } \\
\hline $0-1$ & $70(44.9 \%)$ & $33(38.4 \%)$ \\
\hline $2-3$ & $76(48.7 \%)$ & $5 \mathrm{I}(59.3 \%)$ \\
\hline $4-5$ & $10(6.40 \%)$ & $2(2.30 \%)$ \\
\hline \multicolumn{3}{|l|}{$\begin{array}{l}\text { Site of original primary } \\
\text { tumor }\end{array}$} \\
\hline Lung & $28(\mid 7.9 \%)$ & $23(26.7 \%)$ \\
\hline Colorectal & $44(28.2 \%)$ & $16(18.6 \%)$ \\
\hline Prostate & $34(21.8 \%)$ & $18(20.9 \%)$ \\
\hline Breast & $35(22.4 \%)$ & 18 (20.9\%) \\
\hline Cervical & $10(6.40 \%)$ & $8(9.30 \%)$ \\
\hline Other* & $5(3.20 \%)$ & $3(3.50 \%)$ \\
\hline \multicolumn{3}{|l|}{ Diagnosis of TNM stage } \\
\hline I & $6(3.80 \%)$ & II (I2.8\%) \\
\hline II & $5 \mathrm{I}(32.7 \%)$ & $33(38.4 \%)$ \\
\hline III & 99 (63.5\%) & $42(48.8 \%)$ \\
\hline $\begin{array}{l}\text { Time from diagnosis of } \\
\text { primary tumor to } \\
\text { progression }\end{array}$ & $2.3(1.3-3.6)$ & $2.7(1.7-4.1)$ \\
\hline \multicolumn{3}{|l|}{ Metastasis location } \\
\hline \multicolumn{3}{|l|}{ Brain } \\
\hline No & $123(78.8 \%)$ & $63(73.3 \%)$ \\
\hline Yes & $33(21.2 \%)$ & $23(26.7 \%)$ \\
\hline \multicolumn{3}{|l|}{ Bone } \\
\hline No & 70 (44.9\%) & $39(45.3 \%)$ \\
\hline Yes & $86(55.1 \%)$ & 47 (54.7\%) \\
\hline
\end{tabular}

(Continued)
Table I (Continued).

\begin{tabular}{|c|c|c|}
\hline & $\begin{array}{l}\text { Maintenance } \\
\text { Therapy } \\
(n=I 56)\end{array}$ & $\begin{array}{l}\text { Local } \\
\text { Radiotherapy } \\
(n=86)\end{array}$ \\
\hline \multicolumn{3}{|l|}{ Lung } \\
\hline No & $108(69.2 \%)$ & $55(64.0 \%)$ \\
\hline Yes & $48(30.8 \%)$ & $31(36.0 \%)$ \\
\hline \multicolumn{3}{|l|}{ Adrenal } \\
\hline No & $150(96.2 \%)$ & 79 (91.9\%) \\
\hline Yes & $6(3.80 \%)$ & $7(8.10 \%)$ \\
\hline \multicolumn{3}{|l|}{ Liver } \\
\hline No & 118 (75.6\%) & $68(79.1 \%)$ \\
\hline Yes & 38 (24.4\%) & I8 (20.9\%) \\
\hline \multicolumn{3}{|l|}{ Non-region lymph nodes } \\
\hline No & $144(92.3 \%)$ & $82(95.3 \%)$ \\
\hline Yes & $12(7.70 \%)$ & $4(4.70 \%)$ \\
\hline \multicolumn{3}{|l|}{ No. of metastases } \\
\hline $\mathrm{I}-2$ & $88(56.4 \%)$ & $57(66.3 \%)$ \\
\hline $3-5$ & $68(43.6 \%)$ & $29(33.7 \%)$ \\
\hline \multicolumn{3}{|l|}{$\begin{array}{l}\text { Subsequent systemic } \\
\text { treatment }\end{array}$} \\
\hline No & $47(30.1 \%)$ & 30 (34.9\%) \\
\hline Yes & 109 (69.9\%) & $56(65.1 \%)$ \\
\hline \multicolumn{3}{|l|}{ PET-CT } \\
\hline No & $48(30.8 \%)$ & $24(27.9 \%)$ \\
\hline Yes & $108(69.2 \%)$ & $62(72.1 \%)$ \\
\hline \multicolumn{3}{|l|}{$\begin{array}{l}\text { Biopsies at oligometastatic } \\
\text { sites }\end{array}$} \\
\hline No & 149 (95.5\%) & $83(96.5 \%)$ \\
\hline Yes & 7 (4.50\%) & $3(3.50 \%)$ \\
\hline
\end{tabular}

Notes: *=Larynx $(n=2)$, Pancreatic $(n=3)$, Stomach $(n=2)$, Liver $(n=1)$.

Abbreviations: ECOG PS, Eastern Cooperative Oncology Group Performance Status; COPD, Chronic Obstructive Pulmonary Disease; PET-CT, Positron Emission Tomography Computed Tomography.

months (95\% CI: 12.8-29.2) for patients treated with LRTOS and 14 months (95\% CI: 11.4-16.6) for those treated without local radiotherapy $(\mathrm{p}=0.001$, Figure 3 ).

\section{Univariate and Multivariate Analyses of OS}

In the univariate analysis, LRTOS (HR $=0.45,95 \% \mathrm{CI}$ : $0.35-0.61, \mathrm{p}<0.001$ ), good ECOG PS (HR $=0.53,95 \%$ CI: $0.40-0.70, p<0.001)$, earlier diagnosis of TNM stage $(p=0.047)$, longer interval from diagnosis of primary tumor to progression $(\mathrm{HR}=0.83,95 \% \mathrm{CI}: 0.76-0.92$, $\mathrm{p}<0.001)$, subsequent systemic treatment $(\mathrm{HR}=0.56$, 95\% CI: $0.41-0.77, \mathrm{p}<0.001$ ), and $1-2$ metastases (HR $=$ $0.67,95 \%$ CI: $0.51-0.89, \mathrm{p}=0.006)$ were associated with a better OS (Table 3, Figure 4). 
Table 2 Local Radiotherapy for All Oligometastatic Sites Regimen

\begin{tabular}{|c|c|c|}
\hline $\begin{array}{l}\text { Sites of Disease/Treatment } \\
\text { Regimen }\end{array}$ & Patients & $\begin{array}{c}\text { No. of Patients } \\
\text { (\%) }\end{array}$ \\
\hline Brain & 23 & \\
\hline Dt $50 \%$ Isodose line $22 \mathrm{~Gy} / \mathrm{I} \mathrm{f}^{*}$ & 8 & $34.8 \%$ \\
\hline Dt 60Gy/20 f & 6 & $26.1 \%$ \\
\hline Dt 50Gy/20 f+WBRT(40Gy/20f) & 4 & $17.4 \%$ \\
\hline Dt 30Gy/lof (WBRT) & 5 & $21.7 \%$ \\
\hline Bone & 47 & \\
\hline Dt 30Gy/lof & 12 & $25.5 \%$ \\
\hline Dt 40Gy/20 f & 28 & $59.6 \%$ \\
\hline Dt 60Gy/30 f & 7 & $14.9 \%$ \\
\hline Lung & 31 & \\
\hline $\begin{array}{l}\text { Dt } 70 \% \text { Isodose line } 70-78 \mathrm{~Gy} / \\
10-14 \mathrm{f}^{\#}\end{array}$ & 10 & $32.2 \%$ \\
\hline Dt 70Gy/I5 f & 8 & $25.8 \%$ \\
\hline Dt 70Gy/30 f & I & $3.2 \%$ \\
\hline Dt 60Gy/20 f & 7 & $22.6 \%$ \\
\hline Dt 60Gy/25 f & 5 & $16.2 \%$ \\
\hline Liver & 18 & \\
\hline $\begin{array}{l}\text { Dt } 70 \% \text { Isodose line } 70-78 \mathrm{~Gy} / \\
10-14 f^{\#}\end{array}$ & 8 & $44.5 \%$ \\
\hline Dt $65 \mathrm{~Gy} / 20 \mathrm{f}$ & 2 & $11.1 \%$ \\
\hline Dt 60Gy/I5 f & 2 & $11.1 \%$ \\
\hline Dt 45Gy/I5 f & 6 & $33.3 \%$ \\
\hline Adrenal & 7 & \\
\hline Dt $70 \%$ Isodose line $70 \mathrm{~Gy} / \mathrm{I}$ of & 4 & $57.1 \%$ \\
\hline Dt 70Gy/I5 f & I & $14.3 \%$ \\
\hline Dt 60Gy/20 f & I & $14.3 \%$ \\
\hline Dt 45Gy/I5 f & I & $14.3 \%$ \\
\hline Non-region lymph nodes & 4 & \\
\hline Dt 70Gy/35 f & I & $25.0 \%$ \\
\hline $\mathrm{Dt} 60 \mathrm{~Gy} / 30 \mathrm{f}$ & 2 & $50.0 \%$ \\
\hline Dt $50 \mathrm{~Gy} / 25 \mathrm{f}$ & I & $25.0 \%$ \\
\hline
\end{tabular}

Notes: *The Head Gamma Knife; ${ }^{\#}$ The Body Gamma Knife.

Abbreviations: Dt, Dose of Target; WBRT, Whole-Brain Radiation Therapy.

Multivariate Cox regression analysis indicated that LRTOS (HR $=0.49,95 \%$ CI: $0.35-0.67, \mathrm{p}<0.001)$, good ECOG PS (HR $=0.69,95 \%$ CI: $0.49-0.97, \mathrm{p}=$ 0.032 ), longer interval from diagnosis of primary tumor to progression ( $\mathrm{HR}=0.87,95 \% \mathrm{CI}$ : $0.78-0.97, \mathrm{p}=0.015$ ), and subsequent systemic treatment $(\mathrm{HR}=0.52,95 \% \mathrm{CI}$ : $0.38-0.72, \mathrm{p}<0.001)$ were independent prognostic factors for better OS (Table 3, Figure 5).

To further identify patient subgroups that might benefit from LRTOS, patients were subdivided based on the type of the primary tumor. The mOS rates were significantly improved following application of LRTOS for lung cancer (22 vs 15 months, HR $=0.34,95 \%$ CI: $0.15-0.75$, p $=$ 0.006), colorectal cancer (20 vs 14 months, $H R=0.22$,
95\% CI: $0.10-0.49, \mathrm{p}<0.001$ ), prostate cancer (42 vs 25 months, HR $=0.22,95 \%$ CI: $0.16-0.65, \mathrm{p}=0.002)$, and breast cancer ( 31 vs 18 months, HR $=0.26,95 \%$ CI: 0.12 $0.55, \mathrm{p}<0.001$ ) (Figure 6).

Univariate analysis of OS for patients without subsequent systemic treatment revealed that LRTOS (HR = $0.42,95 \%$ CI: $0.24-0.73, \mathrm{p}<0.001)$ and good ECOG PS $(\mathrm{HR}=0.54,95 \% \mathrm{CI}: 0.32-0.90, \mathrm{p}=0.018)$ correlated with better OS. Further multivariate analysis showed that LRTOS was the only independent prognostic factor for better OS (HR $=0.47,95 \%$ CI: $0.26-0.83, \mathrm{p}=0.010)$ (Table 4).

\section{Toxicity}

The most common adverse reactions were fatigue, leukopenia, radiation-induced pneumonitis, thrombocytopenia, and esophagitis. Majority of patients experienced grade 1-2 toxicities, and no treatment-related death was recorded. Grade 3 radiation-induced pneumonitis $(\mathrm{n}=3$, $3.5 \%$ ) was found in two patients treated with palivizumab, and in one patient with severe chronic obstructive pulmonary disease. Grade 3 leukopenia $(\mathrm{n}=1,1.2 \%)$ only occurred in one patient who received carboplatin-based chemotherapy. Grade 2 pulmonary fibrosis only occurred in patients treated with a body gamma knife ( $70 \%$ isodose line $70 \mathrm{~Gy} / 10 \mathrm{f}$ ), as shown in Table 5.

\section{Discussion}

Generally, maintaining or improving the quality of life is often the primary goal of treatment decisions for elderly patients with metastatic diseases, whereas prolonging survival is the second. ${ }^{18}$ Despite the remarkable advances of modern antitumor drugs, the occurrence of drug resistance often leads to failure of treatment in patients with metastatic tumors. Meanwhile, many elderly patients cannot receive guideline-recommended intensive curative treatment due to an unacceptable risk of treatment-related adverse effects; besides, the patients refuse any recommended systemic therapy. ${ }^{19}$ In the present study, median age of the patients was 70 years and $57.4 \%$ of patients had two or more chronic conditions. Further, $13 \%$ of the patients underwent guideline-recommended intensive systemic treatment and 77 patients only received palliative symptomatic supportive treatment. Palliative radiotherapy is an effective and safe treatment modality for elderly patients with metastatic diseases, relieving pain symptoms. However, the treatment modality for patients with metastatic diseases dramatically changed with inception of the 


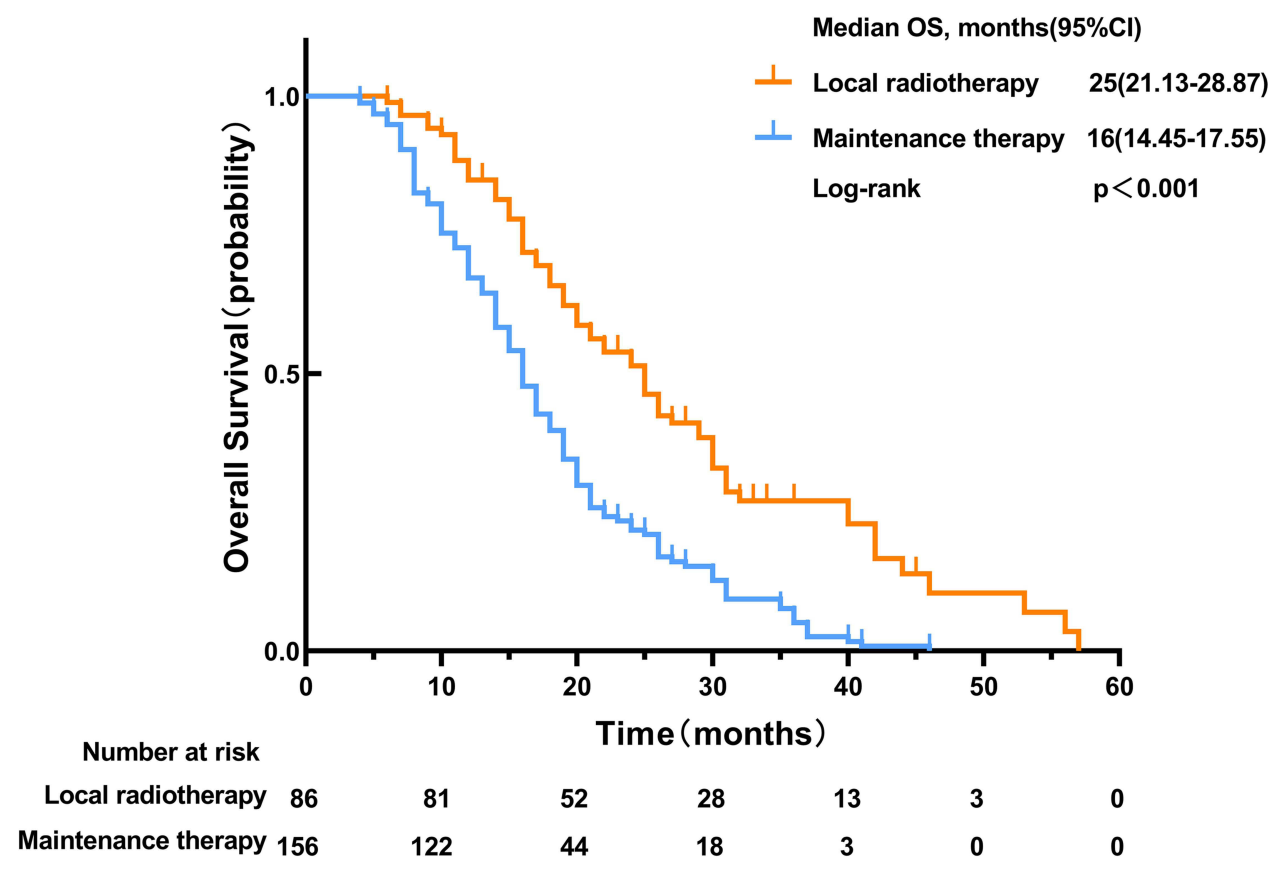

Figure 2 Overall survival of patients in the local radiotherapy and maintenance therapy groups.

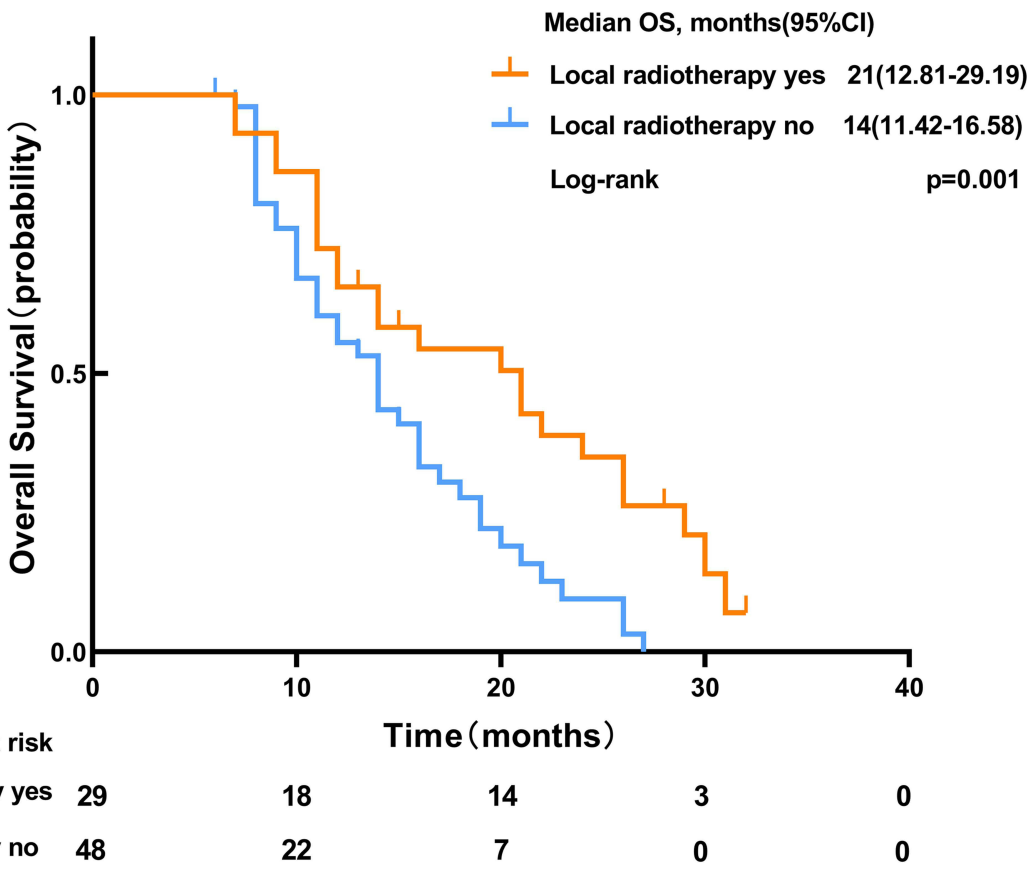

Figure 3 Overall survival of patients who received local radiotherapy at all oligometastatic sites and those who did not receive subsequent systemic treatment.

new concept of "oligometastatic disease". 7,13,17 Results of the current study showed that group treated with the local radiotherapy had better survival as compared with the maintenance therapy. Similarly, those treated with LRTOS had a significantly prolonged mOS in the subgroup of patients without subsequent systemic treatment as compared to patients treated without local radiotherapy (21 vs 14 months, $\mathrm{p}=0.001$ ).

The ideal approach to treating oligometastatic disease remains controversial, and synchronous as well as metachronous oligometastatic diseases show different prognoses. Gomez et $\mathrm{al}^{20}$ conducted the first multicenter, 
Table 3 Factors Associated with Overall Survival in Univariate and Multivariate Analyses

\begin{tabular}{|c|c|c|c|c|c|c|}
\hline \multirow[t]{2}{*}{ Variable } & \multicolumn{3}{|c|}{ Univariable } & \multicolumn{3}{|c|}{ Multivariable } \\
\hline & HR & $95 \% \mathrm{Cl}$ & $\mathbf{P}$ & HR & $95 \% \mathrm{Cl}$ & $\boldsymbol{P}$ \\
\hline \multicolumn{7}{|l|}{ Gender } \\
\hline Male vs Female & 0.77 & $0.58-1.01$ & 0.060 & & & \\
\hline Age & 0.99 & $0.96-1.16$ & 0.134 & & & \\
\hline \multicolumn{7}{|l|}{ EOCG performance status } \\
\hline $0-1$ vs 2 & 0.53 & $0.40-0.70$ & $<0.001$ & 0.69 & $0.49-0.97$ & 0.032 \\
\hline \multicolumn{7}{|l|}{ Smoking status } \\
\hline Present or former smoker vs.Nonsmoker & 1.13 & $0.86-1.48$ & 0.396 & & & \\
\hline Educational degree & & & 0.178 & & & \\
\hline \multicolumn{7}{|l|}{ Elementary school } \\
\hline Junior or senior high school & 0.76 & $0.56-1.03$ & 0.083 & & & \\
\hline Undergraduate & 0.69 & $0.38-1.23$ & 0.207 & & & \\
\hline \multicolumn{7}{|l|}{ No. of underlying disease } \\
\hline \multicolumn{7}{|l|}{$0-1$} \\
\hline $2-3$ & 1.14 & $0.86-1.52$ & 0.366 & & & \\
\hline $4-5$ & 0.76 & $0.4 I-I .4 I$ & 0.387 & & & \\
\hline \multicolumn{7}{|l|}{ Diagnosis of TNM stage } \\
\hline \multicolumn{7}{|l|}{ I } \\
\hline II & 1.78 & $0.89-3.56$ & 0.106 & & & \\
\hline III & 2.18 & $1.10-4.29$ & 0.025 & & & \\
\hline \multicolumn{7}{|l|}{ progression } \\
\hline \multicolumn{7}{|l|}{ No. of metastases } \\
\hline $\mathrm{I}-2$ vs $3-5$ & 0.67 & $0.51-0.89$ & 0.006 & & & \\
\hline \multicolumn{7}{|l|}{ Subsequent systemic treatment } \\
\hline Yes vs No & 0.56 & $0.4 I-0.77$ & $<0.001$ & 0.52 & $0.38-0.72$ & $<0.001$ \\
\hline \multicolumn{7}{|l|}{ Local radiotherapy for all OS } \\
\hline Yes vs No & 0.45 & $0.35-0.61$ & $<0.001$ & 0.49 & $0.35-0.67$ & $<0.001$ \\
\hline
\end{tabular}

Abbreviations: ECOG PS, Eastern Cooperative Oncology Group Performance Status; OS, Oligometastatic Sites.

randomized controlled trial in 49 patients with synchronous oligometastatic non-small-cell lung cancer without progression after first-line systemic therapy. Results of the study showed that local consolidative therapy (radiotherapy or resection of all lesions) significantly improved progression-free survival (PFS) as compared with maintenance therapy alone (11.9 vs 3.9 months, $p=0.0054)$. However, the endpoint of this study was PFS.

A separate study $^{13}$ analyzed longer-term OS rates and found that local consolidative therapy significantly prolonged the OS as compared with maintenance therapy or observation (41.2 vs 18.9 months, $\mathrm{p}=0.017$ ). The described results demonstrated that definitive local therapy for all oligometastatic sites can delay disease progression and translate into a survival benefit in patients with synchronous oligometastases. According to Palma et al, ${ }^{17}$ patients with MOC (breast, lung, colorectal, and prostate cancer) and having controlled primary tumors could benefit from stereotactic body radiotherapy to all oligometastatic sites as compared with the standard of care palliative treatment (41 vs 28 months, $\mathrm{p}=0.009$ ). Elsewhere, $\mathrm{Li}$ et $\mathrm{al}^{21}$ reported that radiotherapy is a feasible and favorable treatment for patients with metachronous oligometastatic esophageal cancer, and a radical radiation dose with BED10 $\geq 60$ Gy has important benefits in extending survival of the patient.

It is evident that all the above studies performed radical local treatment for primary tumors and all oligometastatic sites. Patients with oligometastatic disease had a survival gain from a high rate of local tumor and metastasis control. Several studies showed a high local control rate in hypofractionated radiotherapy $\left(\mathrm{BED}_{10} \geq 100 \mathrm{~Gy}\right)$ lesions. ${ }^{22,23}$ Sharma et $\mathrm{al}^{24}$ reported that SBRT to pulmonary oligometastases was associated with a 2-year OS of $63 \%$. In our study, $60.9 \%(14 / 23)$ of patients with brain metastases received radical local radiotherapy (50\% isodose line $22 \mathrm{~Gy} / 1 \mathrm{f}=8$, 
A

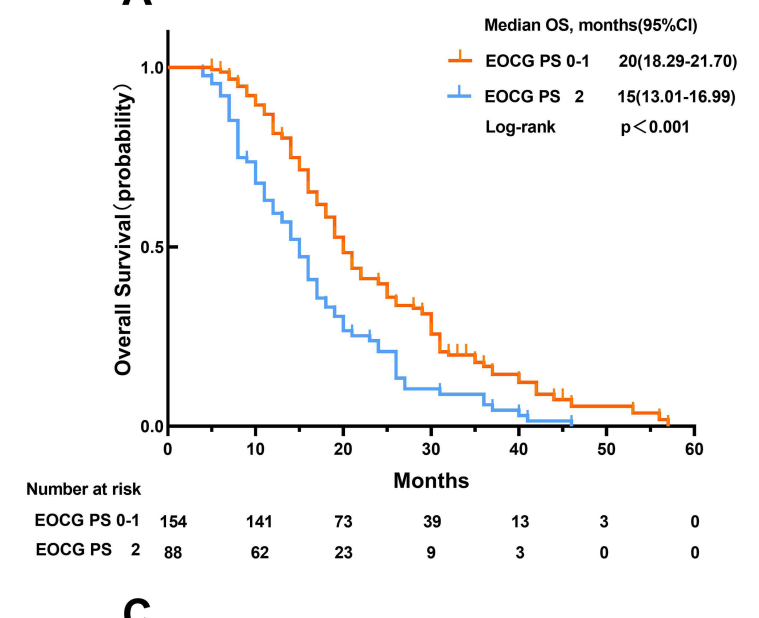

C

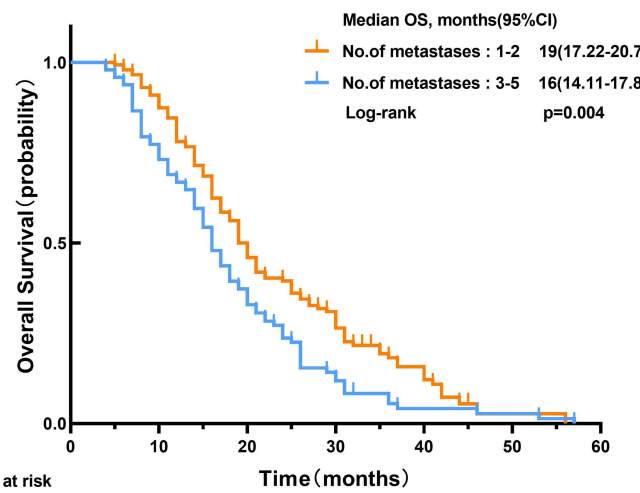

B

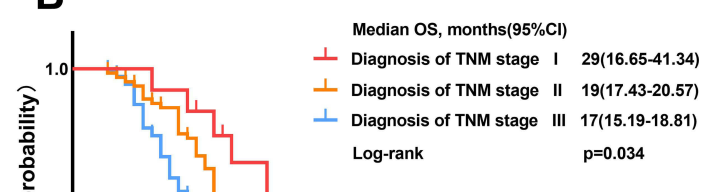

Number at risk

Diagnosis of TNM stage

Diagnosis of TNM stage II 84

Diagnosis of TNM stage III 141

D

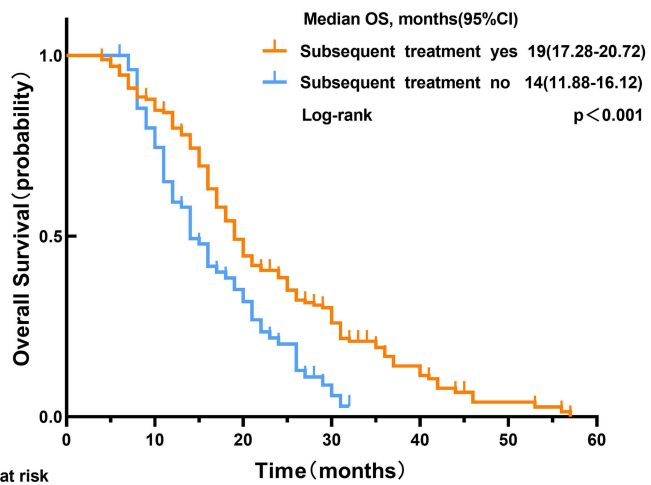

Number at risk

$\begin{array}{llllllll}\text { Subsequent treatment yes } 165 & 144 & 75 & 43 & 16 & 4 & 0\end{array}$

Subsequent treatment no 77

$59 \quad 21$

Figure 4 Overall survival associated with ECOG PS 0-I vs 2 (A), diagnosis of TNM stages I, II, and III (B), number of metastases I-2 vs 3-5 (C), and subsequent treatment yes vs no (D).

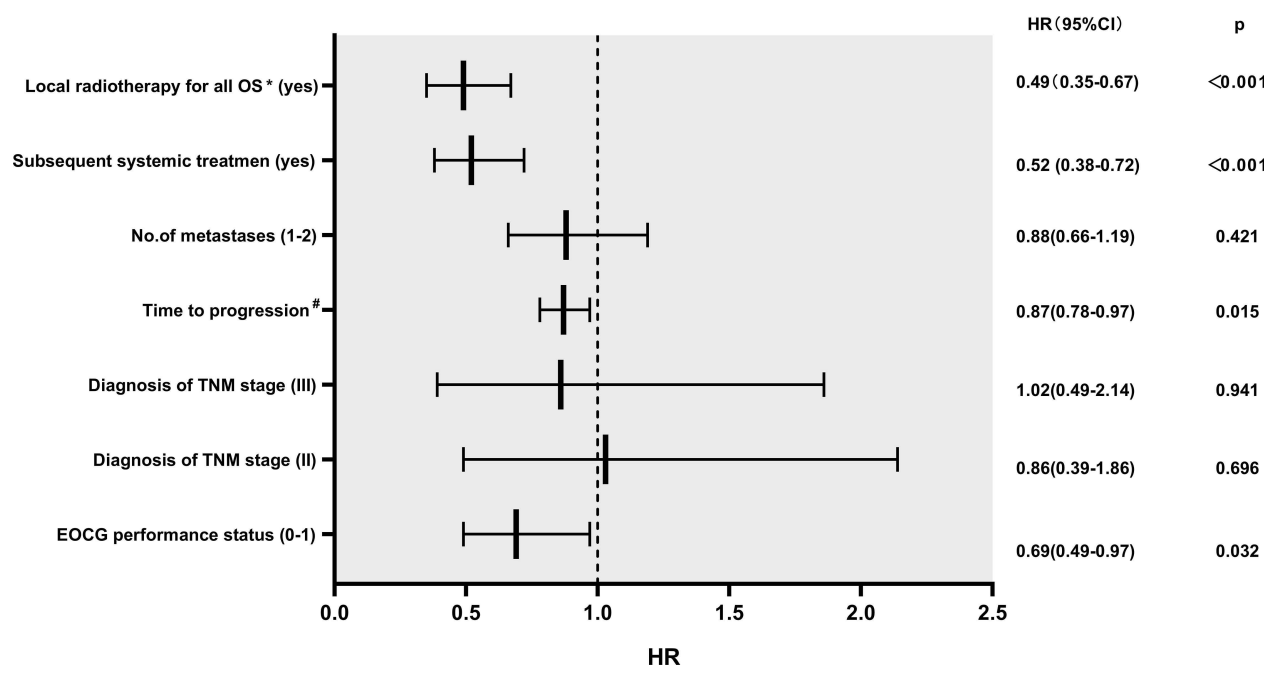

Figure 5 Multivariate analysis of the prognosis of elderly patients with metachronous oligometastatic cancer. OS*= oligometastatic sites, time to progression ${ }^{\#}=$ time from diagnosis of the primary tumor to progression. 

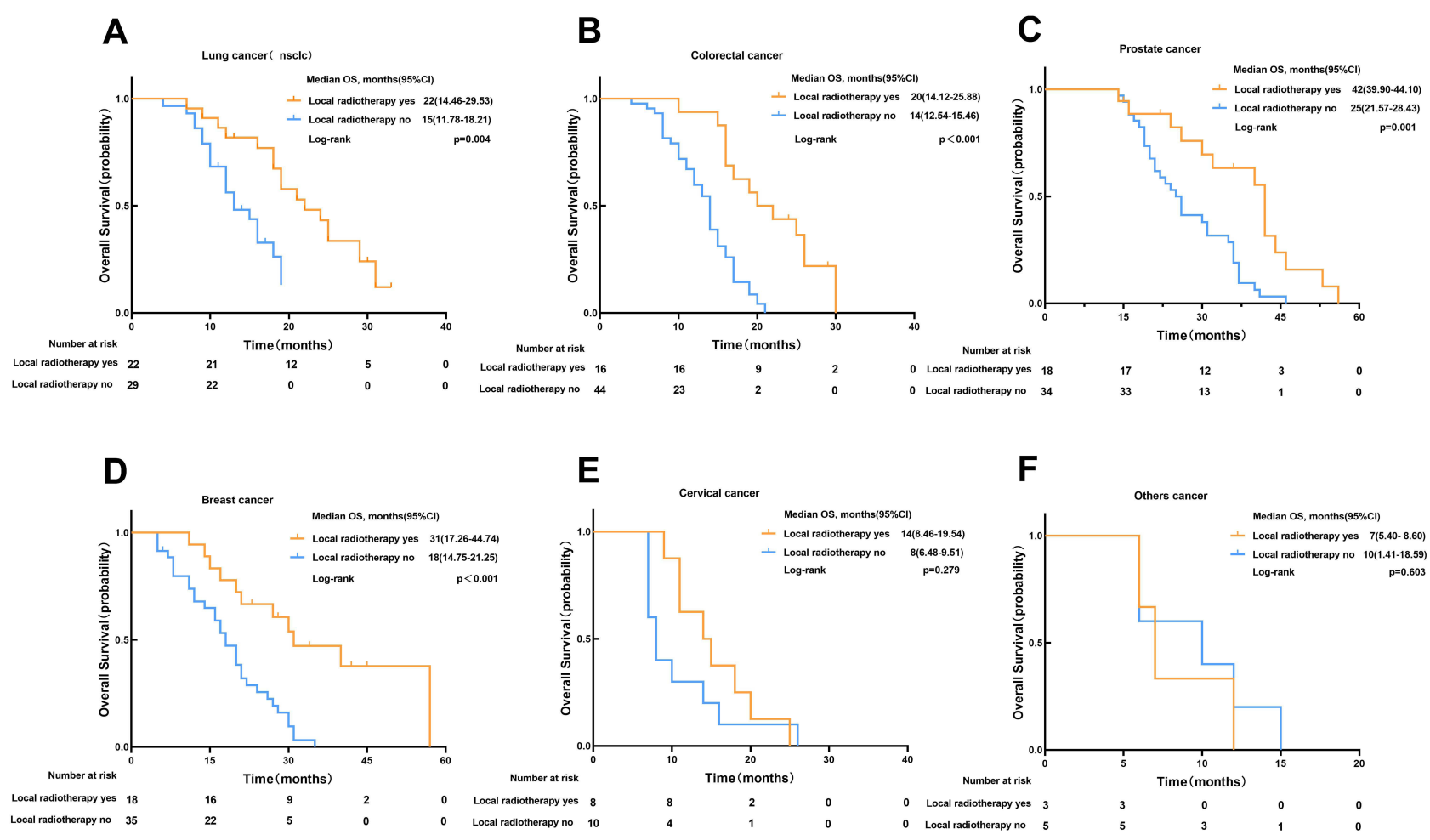

Figure 6 Overall survival for each type of primary tumor. Lung cancer (A), colorectal cancer (B), prostate cancer (C), breast cancer (D), cervical cancer (E), and other cancers $(\mathbf{F})$. Other cancers = larynx cancer $(n=2)$, pancreatic cancer $(n=3)$, stomach cancer $(n=2)$, liver cancer $(n=1)$.

$60 \mathrm{~Gy} / 20 \mathrm{f}=6), 58 \%(18 / 31)$ of patients with lung metastases received hypo-fractionated radiotherapy $(70 \%$ isodose line $70-78 \mathrm{~Gy} / 10-14 \mathrm{f}=10,70 \mathrm{~Gy} / 15 \mathrm{f}=8), 44.5 \%(8 / 18)$ of patients with liver metastases were treated with a body gamma knife (70\% isodose line 70-78 Gy/10-14f), and $57.1 \%(4 / 7)$ of patients with adrenal metastases received a body gamma knife (70\% isodose line $70 \mathrm{~Gy} / 10 \mathrm{f})$. Although gamma knife fractionated stereotactic radiotherapy has unique advantages for treating multiple tumors, it remains unfamiliar to most radiotherapy doctors. This unique dose distribution approach suits elderly patients and is suitable for tumors with relatively regular shapes. ${ }^{25,26}$ Therefore, our study might be biased in selecting patients treated with gamma knife fractionated stereotactic radiotherapy. The optimal local radiotherapy regimen of the oligometastatic disease depends on several factors, including tumor location, tumor size, and tumor shape. Further Phase III studies are needed.

The current scarcity of biomarkers has made imaging the most relevant diagnostic tool for defining oligometastatic disease. ${ }^{27}$ The PET-CT has a higher sensitivity and specificity than other diagnostic modalities in detecting small metastases. ${ }^{28}$ In the current study, 8 patients were diagnosed with non-regional lymph node metastasis using
PET-CT (4 patients performed pathological biopsy), but no tumor was evident in CT or MRI. However, only $70.2 \%$ (n $=170$ ) of patients performed PET-CT examination. This was due to the medical insurance does not cover the expense of PET-CT, and PET-CT is more expensive than CT and MRI. Some studies showed that microRNA profiles correlated with progression or differentiated between oligometastatic and polymetastatic disease. ${ }^{29,30}$ Accurate biomarkers for the diagnosis of oligometastatic diseases are urgently needed. In the current study, 3 pancreatic cancer patients with isolated liver metastases received radical local radiotherapy to liver metastases. The survival outcomes suggested that patients with low CA199 levels could benefit from radical local radiotherapy to liver metastases as compared with those with high CA199 levels (15 vs 9 months). It may be hypothesized that imaging combined with specific biomarkers is beneficial for diagnosing and treating oligometastatic disease.

The choice of optimal systemic therapy is especially unclear in metachronous oligometastatic cancers, especially in elderly patients. In the present study, most elderly patients did not receive a guideline-recommended standard course of systemic treatment due to various factors, including underlying diseases, infections, advanced age, and geriatric 
Table 4 Factors Associated with Overall Survival in Univariate and Multivariate Analyses in Patients without Subsequent Systemic Treatment

\begin{tabular}{|c|c|c|c|c|c|c|}
\hline \multirow[t]{2}{*}{ Variable } & \multicolumn{3}{|c|}{ Univariable } & \multicolumn{3}{|c|}{ Multivariable } \\
\hline & HR & $95 \% \mathrm{Cl}$ & $\mathbf{P}$ & HR & $95 \% \mathrm{Cl}$ & $\boldsymbol{P}$ \\
\hline \multicolumn{7}{|l|}{ Gender } \\
\hline Male vs Female & 0.69 & $0.42-1.14$ & 0.152 & & & \\
\hline Age & 0.99 & $0.95-1.04$ & 0.967 & & & \\
\hline \multicolumn{7}{|l|}{ EOCG performance status } \\
\hline $0-I$ vs 2 & 0.54 & $0.32-0.90$ & 0.018 & & & \\
\hline \multicolumn{7}{|l|}{ Smoking status } \\
\hline Present or former smoker vs. & 0.76 & $0.46-1.25$ & 0.285 & & & \\
\hline \multicolumn{7}{|l|}{ Nonsmoker } \\
\hline Educational degree & & & 0.444 & & & \\
\hline \multicolumn{7}{|l|}{ Elementary school } \\
\hline Junior or senior high school & 0.95 & $0.52-1.74$ & 0.877 & & & \\
\hline Undergraduate & 1.751 & $0.63-4.89$ & 0.285 & & & \\
\hline \multicolumn{7}{|l|}{ No. of underlying disease } \\
\hline \multicolumn{7}{|l|}{$0-1$} \\
\hline $2-3$ & 1.32 & $0.80-2.22$ & 0.279 & & & \\
\hline $4-5$ & 1.01 & $0.24-4.32$ & 0.984 & & & \\
\hline \multicolumn{7}{|l|}{ Diagnosis of TNM stage } \\
\hline \multicolumn{7}{|l|}{ I } \\
\hline II & 1.20 & $0.4 I-3.54$ & 0.741 & & & \\
\hline III & 2.02 & $0.72-5.68$ & 0.180 & & & \\
\hline \multicolumn{7}{|l|}{ tumor to progression } \\
\hline \multicolumn{7}{|l|}{ No. of metastases } \\
\hline $\mathrm{I}-2$ vs $3-5$ & 0.78 & $0.47-1.29$ & 0.328 & & & \\
\hline \multicolumn{7}{|l|}{ Local radiotherapy for all OS } \\
\hline Yes vs No & 0.42 & $0.24-0.73$ & 0.002 & 0.47 & $0.26-0.83$ & 0.010 \\
\hline
\end{tabular}

Abbreviations: ECOG PS, Eastern Cooperative Oncology Group Performance Status; OS, Oligometastatic Sites.

Table 5 Acute and Long-Term Toxicities in the Local Radiotherapy Group

\begin{tabular}{|l|l|l|l|}
\hline CTCAEv5.0 & Grade I No. (\%) & Grade 2 No. (\%) & Grade 3 No. (\%) \\
\hline Acute toxicities & & & \\
Skin rash & $13(15.1 \%)$ & $5(5.8 \%)$ & $3(3.5 \%)$ \\
Pneumonia* & $14(16.3 \%)$ & $5(5.8 \%)$ & 0 \\
Fatigue & $35(40.7 \%)$ & $13(15.1 \%)$ & 0 \\
Anemia & $12(13.9 \%)$ & $8(9.3 \%)$ & $1(1.2 \%)$ \\
Leukopenia & $20(23.3 \%)$ & $10(11.6 \%)$ & 0 \\
Thrombocytopenia & $11(12.8 \%)$ & $5(5.8 \%)$ & 0 \\
Esophagitis & $10(11.6 \%)$ & $1(1.2 \%)$ & 0 \\
Long-term toxicities & $2(2.3 \%)$ & $1(1.2 \%)$ & 0 \\
Pulmonary fibrosis & $1(1.2 \%)$ & 0 & \\
Pleural effusion & & & \\
\hline
\end{tabular}

Note: $*=$ radiation-induced pneumonitis.

syndromes. Univariate and multivariate analyses demonstrated that systemic treatment was an independent prognostic predictor for survival. This indicated that systemic treatment was an indispensable part of the treatment of oligometastatic cancers. However, this modality must be cautiously considered on an individual basis in elderly 
patients. Over the past few years, significant advances have been made in cancer immunologic therapies, which have been applied as a first-line treatment for some metastatic tumors. Bauml et $\mathrm{al}^{31}$ reported that pembrolizumab after locally ablative therapy for oligometastatic non-small cell lung cancer improved PFS with no reduction in quality of life (median PFS: 19.1 months). Recent studies ${ }^{32,33}$ have shown a synergic effect of radiotherapy combined with immune checkpoint inhibitors, especially SBRT irradiation to the tumor. In our study, 22 patients received programmed cell death-1 (PD-1) or programmed cell death-ligand 1 (PDL1) immunotherapy, of which 10 combined with LRTOS. Patients treated with a combination of immunotherapy with local radiotherapy had longer OS than those treated with immunotherapy alone (17 vs 10 months). Notably, the sample size was too small for further analysis.

This study had some shortcomings. First, the small sample size made it difficult to detect a statistical difference in some subgroups. Second, this study was a retrospective study; hence, a selection bias was inevitable. A prospective randomized controlled study is warranted to validate our findings. Meanwhile, this study was not a multicenter study. Patients treated with gamma knife fractionated stereotactic radiotherapy were obtained from Air Force General Hospital. This is due to the lack of gamma knife equipment in the Beijing Geriatric Hospital. Third, an accurate and continuous systemic treatment regimen could not be reliably ascertained because several patients received systemic therapy at other medical institutions during the follow-up period. Finally, the lack of patient-reported outcome measures (PROMs), geriatric assessment tools, and quality of life (QoL) assessment further limits the study.

\section{Conclusion}

In conclusion, this retrospective study shows that LRTOS may improve the survival of elderly patients with MOC. The optimal combination of systemic treatment and local radiotherapy for elderly MOC patients need to be further explored.

\section{Abbreviations}

LRTOS, local radiotherapy to all oligometastatic site; MOC, metachronous oligometastatic cancers; mOS, median overall survival; CI, confidence interval; ECOG PS, Eastern Cooperative Oncology Group Performance Status; MRI, magnetic resonance imaging; PET-CT, positron emission tomography computed tomography; 3DCRT, conformal radiotherapy; IMRT, intensity-modulated radiation therapy;
SBRT, stereotactic body radiotherapy; SRS, stereotactic radiosurgery; WBRT, whole-brain radiation therapy; CTCAE, Common Terminology Criteria for Adverse Events; BED, biologically effective dose; PD-1, programmed cell death-1; PD-L1, programmed cell death-ligand 1; NSCLC, non-small cell lung cancer; CGA, comprehensive geriatric assessment.

\section{Ethical Statement}

The authors are accountable for all aspects of the work in ensuring that questions related to the accuracy or integrity of any part of the work are appropriately investigated and resolved. The study was conducted according to the Declaration of Helsinki (as revised in 2013) and was approved by The Ethics Committee of Beijing Geriatric Hospital. For this retrospective study, the patients were included in this study only after obtaining written informed consent.

\section{Funding}

Supported by Beijing Geriatric Hospital Special Fund for Geriatrics Research (2020bjlnyy-q-1).

\section{Disclosure}

The authors have declared no conflicts of interest.

\section{References}

1. Reese ES, Onukwugha E, Hanna N, Seal BS, Mullins CD. Clinical and demographic characteristics associated with the receipt of chemotherapy treatment among 7951 elderly metastatic colon cancer patients. Cancer Med. 2013;2(6):907-915. doi:10.1002/cam4.143

2. Reesink DJ, van de Garde EMW, Peters,BJM, et al. Treatment patterns and clinical outcomes of chemotherapy treatment in patients with muscle-invasive or metastatic bladder cancer in the Netherlands. Sci Rep. 2020;10(1):15822. doi:10.1038/s41598-020-72820-y

3. Feliu J, Heredia-Soto V, Gironés R, et al. Management of the toxicity of chemotherapy and targeted therapies in elderly cancer patients. Clin Transl Oncol. 2020;22(4):457-467. doi:10.1007/s12094-019-02167-y

4. Wang S, Wong ML, Hamilton N, Davoren JB, Jahan TM, Walter LC. Impact of age and comorbidity on non-small-cell lung cancer treatment in older veterans. $J$ Clin Oncol. 2012;30(13):1447-1455. doi:10.1200/JCO.2011.39.5269

5. Hellman S, Weichselbaum RR. Oligometastases. J Clin Oncol. 1995;13(1):8-10. doi:10.1200/JCO.1995.13.1.8

6. Dingemans AC, Hendriks LEL, Berghmans T, et al. Definition of synchronous oligometastatic non-small cell lung cancer-a consensus report. J Thorac Oncol. 2019;14(12):2109-2119. doi:10.1016/j.jtho.2019.07.025

7. Palma DA, Salama JK, Lo SS, et al. The oligometastatic state separating truth from wishful thinking. Nat Rev Clin Oncol. 2014;11 (9):549-557. doi:10.1038/nrclinonc.2014.96

8. Kim JH, Rha SY, Kim C, et al. Clinicopathologic features of metachronous or synchronous gastric cancer patients with three or more primary sites. Cancer Res Treat. 2010;42(4):217-224. doi:10.4143/ crt.2010.42.4.217

9. Mekenkamp LJ, Koopman M, Teerenstra S, et al. Clinicopathological features and outcome in advanced colorectal cancer patients with synchronous vs. metachronous metastases. Br J Cancer. 2010;103 (2):159-164. doi:10.1038/sj.bjc.6605737 
10. Guckenberger M, Lievens Y, Bouma AB, et al. Characterisation and classification of oligometastatic disease: a European society for radiotherapy and oncology and European organisation for research and treatment of cancer consensus recommendation. Lancet Oncol. 2020;21(1):e18-e28. doi:10.1016/S1470-2045(19)30718-1

11. Chalkidou A, Macmillan T, Grzeda MT, et al. Stereotactic ablative body radiotherapy in patients with oligometastatic cancers: a prospective, registry-based, single-arm, observational, evaluation study. Lancet Oncol. 2021;22(1):98-106. doi:10.1016/S14702045(20)30537-4

12. Iyengar P, Wardak Z, Gerber DE, et al. Consolidative radiotherapy for limited metastatic non-small-cell lung cancer: a phase 2 randomized clinical trial. JAMA Oncol. 2018;4(1):e173501. doi:10.1001/ jamaoncol.2017.3501

13. Gomez DR, Tang C, Zhang J, et al. Local consolidative therapy vs. maintenance therapy or observation for patients with oligometastatic non-small-cell lung cancer: long-term results of a multi-institutional, phase ii, randomized study. J Clin Oncol. 2019;37(18):1558-1565. doi:10.1200/JCO.19.00201

14. Fleckenstein J, Petroff A, Schäfers HJ, Wehler T, Schöpe J, Rübe C. Long-term outcomes in radically treated synchronous vs. metachronous oligometastatic non-small-cell lung cancer. BMC Cancer. 2016;16:348. doi:10.1186/s12885-016-2379-x

15. Tasca G, Dieci MV, Baretta Z, et al. Synchronous and metachronous breast and ovarian cancer: experience from two large cancer center. Front Oncol. 2020;10:608783. doi:10.3389/fonc.2020.608783

16. Ma CX, Guan X, Wei R, et al. The distinction of clinicopathological characteristics, treatment strategy and outcome in colorectal cancer patients with synchronous vs. metachronous bone metastasis. Front Oncol. 2020;10:974. doi:10.3389/fonc.2020.00974

17. Palma DA, Olson R, Harrow S, et al. Stereotactic ablative radiotherapy versus standard of care palliative treatment in patients with oligometastatic cancers (SABR-COMET): a randomized, phase 2, open-label trial. Lancet. 2019;393(10185):2051-2058. doi:10.1016 S0140-6736(18)32487-5

18. Decoster L, Kenis C, Schallier D, et al. Geriatric assessment and functional decline in older patients with lung cancer. Lung. 2017;195 (5):619-626. doi:10.1007/s00408-017-0025-2

19. Tuca A, Gallego R, Ghanem I, Gil-Raga M, Feliu J. Chemotherapy and targeted agents in the treatment of elderly patients with metastatic colorectal cancer. J Clin Med. 2020;9(12):4015. doi:10.3390/ jem9124015

20. Gomez DR, Blumenschein GR, Lee JJ, et al. Local consolidative therapy versus maintenance therapy or observation for patients with oligometastatic non-small-cell lung cancer without progression after first-line systemic therapy: a multicentre, randomised, controlled, phase 2 study. Lancet Oncol. 2016;17(12):1672-1682. doi:10.1016/ S1470-2045(16)30532-0
21. Li J, Wen Y, Xiang Z, et al. Radical radiotherapy for metachronous oligometastasis after initial treatment of esophageal cancer. Radiother Oncol. 2021;154:201-206. doi:10.1016/j.radonc.2020.09.042

22. Mahadevan A, Blanck O, Lanciano R, et al. Stereotactic body radiotherapy (SBRT) for liver metastasis - clinical outcomes from the international multi-institutional RSSearch ${ }^{\circledR}$ patient registry. Radiat Oncol. 2018;13(1):26. doi:10.1186/s13014-018-0969-2

23. Arcidiacono F, Aristei C, Marchionni A, et al. Stereotactic body radiotherapy for adrenal oligometastasis in lung cancer patients. $\mathrm{Br}$ J Radiol. 2020;93(1115):20200645. doi:10.1259/bjr.20200645

24. Sharma A, Duijm M, Oomen-de Hoop E, et al. Survival and prognostic factors of pulmonary oligometastases treated with stereotactic body radiotherapy. Acta Oncol. 2019;58(1):74-80. doi:10.1080/ 0284186X.2018.1521986

25. Xia T, Li H, Sun Q, et al. Promising clinical outcome of stereotactic body radiation therapy for patients with inoperable Stage I/II non-small-cell lung cancer. Int J Radiat Oncol Biol Phys. 2006;66 (1):117-125. doi:10.1016/j.ijrobp.2006.04.013

26. Li H, Li J, Wang X, et al. Promising clinical outcome with long term follow-up after body Gamma knife stereotactic radiosurgery for patients with early stage non-small cell lung cancer. Front Oncol. 2018;8:618. doi:10.3389/fonc.2018.00618

27. deSouza NM, Tempany CM. A risk-based approach to identifying oligometastatic disease on imaging. Int $J$ Cancer. 2019;144 (3):422-430. doi:10.1002/ijc.31793

28. Liu T, Xu W, Yan WL, Ye M, Bai YR, Huang G. FDG-PET, CT, MRI for diagnosis of local residual or recurrent nasopharyngeal carcinoma, which one is the best? A systematic review. Radiother Oncol. 2007;85(3):327-335. doi:10.1016/j.radonc.2007.11.002

29. Lussier YA, Xing HR, Salama JK, et al. MicroRNA expression characterizes oligometastasis(es). PLoS One. 2011;6(12):e28650. doi:10.1371/journal.pone.0028650

30. Lussier YA, Khodarev NN, Regan K, et al. Oligo- and polymetastatic progression in lung metastasis(es) patients is associated with specific microRNAs. PLoS One. 2012;7:e50141. doi:10.1371/journal. pone.0050141

31. Bauml JM, Mick R, Ciunci C, et al. Pembrolizumab after completion of locally ablative therapy for oligometastatic non-small cell lung cancer: a phase 2 trial. JAMA Oncol. 2019;5(9):1283-1290. doi:10.1001/jamaoncol.2019.1449

32. Butts C, Socinski MA, Mitchell PL, et al. Tecemotide (L-BLP25) versus placebo after chemoradiotherapy for stage III non-small-cell lung cancer (START): a randomised, double-blind, Phase 3 trial. Lancet Oncol. 2014;15(1):59-68. doi:10.1016/S1470-2045(13) 70510-2

33. Herrera FG, Bourhis J, Coukos G. Radiotherapy combination opportunities leveraging immunity for the next oncology practice. $C A$ Cancer J Clin. 2017;67(1):65-85. doi:10.3322/caac.21358
Cancer Management and Research

\section{Publish your work in this journal}

Cancer Management and Research is an international, peer-reviewed open access journal focusing on cancer research and the optimal use of preventative and integrated treatment interventions to achieve improved outcomes, enhanced survival and quality of life for the cancer patient.
The manuscript management system is completely online and includes a very quick and fair peer-review system, which is all easy to use Visit http://www.dovepress.com/testimonials.php to read real quotes from published authors. 